\title{
Time-resolved fluorescence analysis of the mobile flavin cofactor in p-hydroxybenzoate hydroxylase
}

\author{
PETRA A W VAN DEN BERG ${ }^{\mathrm{a}, \mathrm{b}}$ KOERT GREVER, ${ }^{\mathrm{a}}$ ARIE VAN HOEK, ${ }^{\mathrm{b}}$ \\ WILLEM J H VAN BERKEL ${ }^{\mathrm{a}}$ and ANTONIE J W G VISSER ${ }^{\mathrm{a}, \mathrm{b}, \mathrm{c}, *}$ \\ ${ }^{a}$ Laboratory of Biochemistry, Wageningen University, Dreijenlaan 3, 6703 HA, Wageningen, \\ The Netherlands \\ ${ }^{b}$ Microspectroscopy Centre, PO Box 8128, 6700 ET, Wageningen, The Netherlands \\ ${ }^{c}$ Department of Structural Biology, Faculty of Earth and Life Sciences, Vrije Universiteit Amsterdam, \\ De Boelelaan 1085, $1081 \mathrm{HV}$, Amsterdam, The Netherlands \\ e-mail: ton.visser@wur.nl
}

\begin{abstract}
Conformational heterogeneity of the FAD cofactor in $p$-hydroxybenzoate hydroxylase $(\mathrm{PHBH})$ was investigated with time-resolved polarized flavin fluorescence. For binary enzyme/substrate (analogue) complexes of wild-type PHBH and Tyr222 mutants, crystallographic studies have revealed two distinct flavin conformations; the 'in' conformation with the isoalloxazine ring located in the active site, and the 'out' conformation with the isoalloxazine ring disposed towards the protein surface. Fluorescence-lifetime analysis of these complexes revealed similar lifetime distributions for the 'in' and 'out' conformations. The reason for this is twofold. First, the active site of PHBH contains various potential fluorescence-quenching sites close to the flavin. Fluorescence analysis of uncomplexed PHBH Y222V and Y222A showed that Tyr222 is responsible for picosecond fluorescence quenching free enzyme. In addition, other potential quenching sites, including a tryptophan and two tyrosines involved in substrate binding, are located nearby. Since the shortest distance between these quenching sites and the isoalloxazine ring differs only little on average, these aromatic residues are likely to contribute to fluorescence quenching. Second, the effect of flavin conformation on the fluorescence lifetime distribution is blurred by binding of the aromatic substrates: saturation with aromatic substrates induces highly efficient fluorescence quenching. The flavin conformation is therefore only reflected in the small relative contributions of the longer lifetimes.
\end{abstract}

Keywords. Time-resolved fluorescence; flavin; fluorescence lifetime distribution; quenching by electron transfer; enzyme conformations; maximum entropy method.

\section{Introduction}

For most enzymes, conformational dynamics involves mobility of the polypeptide chain. Flavoenzymes in which functionally important motions entail either local conformational changes of the protein backbone (glutathione reductase $\mathrm{e}^{1,2}$ or gross motions of protein domains (thioredoxin reductase ${ }^{3}$ ) have been subject of investigation. In enzymes that contain a prosthetic group such as the flavoenzymes, mobility of the cofactor itself can in principle also play a role in catalysis. A flavoenzyme for which mobility of the isoalloxazine ring of the flavin cofactor has been

\footnotetext{
*For correspondence

Abbreviations: GR: glutathione reductase, MEM: maximum entropy method, PHBH: $p$-hydroxybenzoate hydroxylase
}

shown to be essential for the biological function is $p$-hydroxybenzoate hydroxylase. ${ }^{4,5}$

$p$-Hydroxybenzoate hydroxylase (PHBH) (EC $1 \cdot 14 \cdot 13 \cdot 2$ ) belongs to the class of flavin-dependent monooxygenases. 6 PHBH catalyses the NADPHand $\mathrm{O}_{2}$-dependent conversion of 4-hydroxybenzoate (p-OHB) into 3,4-dihydroxybenzoate, a common intermediate step in the biodegradation of aromatic compounds in soil microorganisms, e.g. in the degradation pathway of lignin. ${ }^{7}$ PHBH is a homodimeric protein containing one molecule of FAD per subunit of $\approx 45 \mathrm{kDa}$. The catalytic properties of the enzyme from Pseudomonas fluorescens and Pseudomonas aeruginosa have been studied extensively with kinetic, spectroscopic and mutagenesis methods. ${ }^{8,9}$ The reaction cycle of PHBH consists of a reductive half-reaction in which the flavin is reduced by NADPH in the presence of bound substrate, and a 


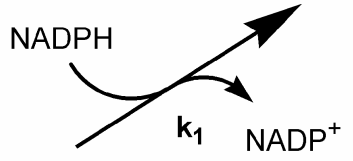<smiles>[R]n1c2nc(=O)[nH]c(=O)c-2nc2cc(C)c(C)cc21</smiles>

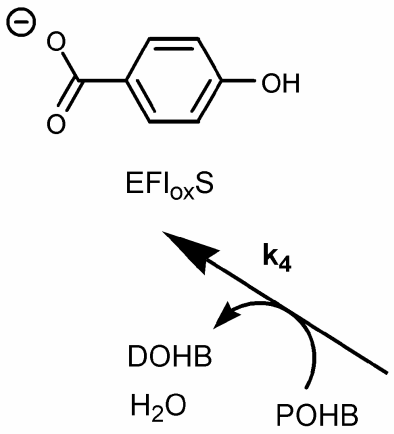<smiles>[R]N1c2cc(C)c(C)cc2Nc2c1n([O-])c(=O)[nH]c2=O</smiles><smiles>O=C([O-])c1ccc(O)cc1</smiles>

$\mathrm{EFI}_{\text {red }} \mathrm{S}$

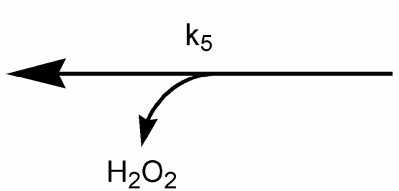<smiles>[R]N1C2=NC(=O)NC(=O)C2(O)Nc2cc(C)c(C)cc21</smiles><smiles>O=C([O-])c1ccc(O)c(O)c1</smiles>

$\mathrm{EFI}_{\mathrm{HOH}^{-}} \mathrm{P}$<smiles>CCC(C)O</smiles><smiles>[R]N1C2=NC(=O)NC(=O)C2(OO)Nc2cc(C)c(C)cc21</smiles><smiles>CCCCOOS</smiles>

Figure 1. Catalytic cycle of $p$-hydroxybenzoate hydroxylase. Rate constants represent; $k_{1}$, reduction of the enzyme-substrate (EFloxS) complex by NADPH; $k_{2}$, formation of flavin (C4a)-hydroperoxide with molecular oxygen; $k_{3}$, substrate hydroxylation; $k_{4}$, dehydration of flavin $(\mathrm{C} 4 \mathrm{a})$-hydroxide and product (3,4-dihydroxybenzoate; DOHB) release, followed by substrate binding ( $p$-OHB); $k_{5}$, uncoupling of hydroxylation through peroxide release.

complex oxidative half-reaction leading to hydroxylation of the substrate (figure 1). In the reductive half-reaction, efficient reduction of the flavin by $\mathrm{NADPH}$ requires a ternary complex in which the substrate $p$-OHB acts as an effector that increases the rate of reduction by about five orders of magnitude, ${ }^{10}$ irrespective of the actual order of binding. The substrate specificity of PHBH is rather narrow and limited by the poor capacity of substrate analogues to stimulate flavin reduction. Site-directed mutagenesis studies have shown that deprotonation of the substrate phenol facilitates reduction. ${ }^{11-13}$ After flavin reduction, $\mathrm{NADP}^{+}$is released, while $p$ OHB remains tightly bound. ${ }^{14}$ In the oxidative halfreaction, molecular oxygen reacts with the reduced enzyme/substrate complex to form a flavin $\mathrm{C} 4 \mathrm{a}$ - hydroperoxide intermediate. Subsequently, in an electrophilic substitution reaction the distal oxygen of the hydroperoxide intermediate is transferred to the substrate yielding the product 3,4-dihydroxybenzoate and a flavin-C4a-hydroxide species. Elimination of water from the flavin-C4a-hydroxide and product release complete the catalytic cycle. NADPH oxidase activity is found in the absence of substrate, or in the presence of non-substrate effectors, where the flavin-C4a-hydroperoxide intermediate decomposes to yield hydrogen peroxide. ${ }^{15}$

For many years, detailed three-dimensional structural information on P. fluorescens PHBH has been available from crystallographic studies of the oxidized and reduced enzyme-substrate and enzyme/product complexes. ${ }^{16-19}$ Binding of $p$-OHB involves inter- 
actions of the carboxyl group with Arg214 (salt bridge $^{20}$ ), Ser212 (hydrogen bonding ${ }^{21,22}$ ) and Tyr222 (hydrogen bonding ${ }^{4,5,23}$ ). The hydroxyl group of the substrate is at hydrogen-bonding distance of Tyr201 and Tyr385. The latter residues have an important function in substrate activation by facilitating substrate deprotonation, and in flavin reduction. ${ }^{11,12}$

In the absence of substrate or in the presence of $\mathrm{NADPH}, \mathrm{PHBH}$ was long found to have poor diffraction properties. ${ }^{24-27}$ Unlike many other pyridine nucleotide-dependent enzymes, PHBH lacks a Rossmann fold for binding NADPH. Based on sitedirected mutagenesis studies, ${ }^{26,28,29}$ an interdomain binding for NADPH was proposed ${ }^{26}$ and a switch in coenzyme specificity was achieved. ${ }^{30}$ Recently, the first crystal structure of PHBH with NADPH was obtained. In this structure of the R220Q mutant, without substrate bound, the NADPH binds in a remarkable conformation, with the nicotinamide moiety pointing away from the flavin. ${ }^{27}$ Such binding mode would require major conformational rearrangements for flavin reduction, most likely both from the flavin and nicotinamide cofactors, as from the polypeptide chain. ${ }^{27}$

The most compelling structural characteristic of $\mathrm{PHBH}$, however, is the mobility of the isoalloxazine ring of the flavin (figure 2). Complexes with the substrate $p$-OHB showed the flavin ring buried in the enzyme active site ('in' conformation). However, complexes with substrate analogues bearing a hydroxyl group at the 2-position (2,4-dihydroxybenzoate, 2-hydroxy-4-aminobenzoate) revealed a conformation in which the flavin ring is located in the cleft that leads to the active site, more towards the surface of the protein ('out' conformation'; see table 1). This 'out' conformation was also found for Y222F PHBH in complex with the natural substrate, ${ }^{5}$ and for the enzyme/substrate complex of wild-type PHBH reconstituted with arabino-FAD. ${ }^{31}$ The observation of two conformations of the flavin explained the distinct differences in flavin absorption perturbation spectra of various binary enzyme/ substrate analogue complexes. ${ }^{5}$ Spectral and kinetic data on the mutant enzymes PHBH Y222A and Y222V supported the role of Tyr222 in flavin mobility. ${ }^{23}$ Based on crystallographic studies, it was proposed that flavin mobility plays a prominent role in catalysis: the 'in' position provides a suitable environment for efficient hydroxylation of the substrate (shielded from the solvent), and the swinging 'out' of the flavin facilitates substrate binding and product release. The 'out' conformation is probably also necessary for efficient flavin reduction, ${ }^{13,26,27,31-34}$ although no conclusive evidence for this has been presented yet. ${ }^{35}$

The three-dimensional structure of substrate-free $\mathrm{PHBH}^{33}$ is nearly identical to that of the enzymesubstrate complex. However, in the free enzyme the flavin ring has increased flexibility and is on average found at a position intermediate between the 'in' and 'out' conformation. In R222Q PHBH, without substrate bound, a similar intermediate position for the flavin was observed. ${ }^{27}$ In this poorly active mutant protein, the FAD domain and substrate binding domain separate slightly to form a cleft that allows solvent access to the active site. Based on

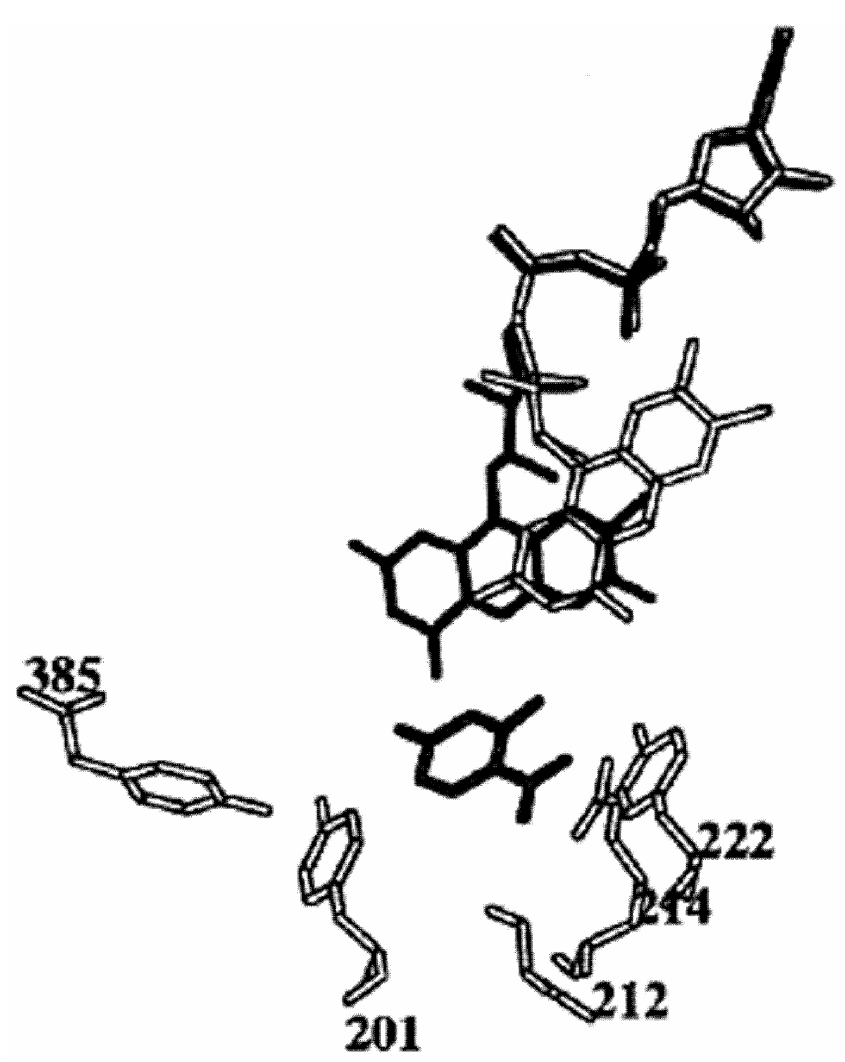

Figure 2. Active site of $\mathrm{PHBH}$ in the 'in' and 'out' conformations. In wild-type PHBH complexed with $p$-OHB, the isoalloxazine ring occupies the 'in' conformation (solid bonds; Schreuder et al 1989). The 'out' conformation (open bonds) is found in the complex with 2,4-dihydroxybenzoate (Schreuder et al 1994), and for several binary enzyme-substrate complexes with Y222 mutant enzymes (Schreuder et al 1994; Gatti et al 1994). An overview of the conformations of the FAD as retrieved from different crystallographic studies is presented in table 1 . 
Table 1. Overview of flavin conformations observed in crystals of uncomplexed $\mathrm{PHBH}$, and in different enzyme/substrate (analogue) complexes.

\begin{tabular}{lcll}
\hline Enzyme & Redox state & \multicolumn{1}{c}{ Substrate analogue } & Conformation \\
\hline PHBH & Ox & - (Free enzyme) & Intermediate $^{\mathrm{a}}$ \\
PHBH & Ox & $p$-OHB & $\mathrm{In}^{\mathrm{b}}$ \\
PHBH & Red & $p$-OHB & $\mathrm{In}^{\mathrm{c}}$ \\
PHBH & Ox & -Aminobenzoate & $\mathrm{In}^{\mathrm{d}}$ \\
PHBH & Ox & 3,4-Dihydroxybezoate & $\mathrm{In}^{\mathrm{e}}$ \\
PHBH & Ox & 2,4-Dihydroxybenzoate & Out $^{\mathrm{d}}$ \\
PHBH & Ox & 2-Hydroxy-4-aminobenzoate & Out $^{\mathrm{d}}$ \\
PHBH Y222F & Ox & $p$-OHB & Out $^{\mathrm{d}}(70 \%)^{\mathrm{f}}$ \\
PHBH Y222A & Ox & 2-Hydroxy-4-aminobenzoate & Out $^{\mathrm{d}}$ \\
\hline
\end{tabular}

${ }^{\mathrm{a}}$ Eppink et al (1999); ${ }^{\mathrm{b}} \mathrm{S}$ Shreuder et al (1989); ${ }^{\circ}$ Schreuder et al (1992); ${ }^{\mathrm{d}} \mathrm{Schreuder}$ et al (1994);

${ }^{\mathrm{e}} \mathrm{Sch}$ reuder et al (1988); ${ }_{\mathrm{f}}^{\mathrm{G}} \mathrm{Gatti}$ et al (1994)

these findings, it has been proposed that concerted protein and flavin motions are needed in the wildtype enzyme to provide a path for $p$-OHB to enter the hydroxylation site. ${ }^{27}$

In this contribution, time-resolved flavin fluorescence studies are discussed that were performed to obtain more insight into the mobility of the flavin in $P$. fluorescens PHBH. Polarized fluorescence decay analyses were focussed on the different microenvironments of the flavin in the 'in' and 'out' conformations. In order to sample different (known) conformations, binary enzyme/substrate analogue complexes of both wild-type PHBH and the mutant Y222V were studied in the oxidized state. The timeresolved fluorescence data are discussed in relation to the mechanisms of flavin fluorescence quenching as reported in previous work. ${ }^{1-3,36,37}$

\section{Materials and methods}

\subsection{Sample preparation}

$p$-Hydroxybenzoate hydroxylase from $P$. fluorescens was isolated from $E$. coli TG2 cells transformed with the expression plasmid pAW45. ${ }^{20}$ The microheterogeneity-resistant mutant PHBH C116S was used as pseudo wild-type enzyme in order to prevent protein aggregation resulting from the oxidation of the cysteine at position 116. ${ }^{12,38-40}$ Residue Cys 116 is located far away from the active site, and its mutation does not influence any of the catalytic properties of the enzyme. Mutant genes encoding for PHBH Y222A and Y222V were constructed as reported earlier. ${ }^{23}$ All enzymes were purified according to the method described ${ }^{20}$ and stored in $80 \%$ ammonium sulphate at $277 \mathrm{~K}$. Directly prior to use, traces of free FAD were removed by chromatography on a Biogel PGD-6 column (Biorad) equilibrated with measuring buffer.

All spectroscopic measurements were carried out in $50 \mathrm{mM}$ potassium phosphate buffer $\mathrm{pH} 7.5$ at $293 \mathrm{~K}$. The enzyme concentration was kept at $10 \mu \mathrm{M}$ with respect to FAD with $\varepsilon_{450 \mathrm{PHBH}}=10 \cdot 2 \mathrm{mM}^{-1} \mathrm{~cm}^{-1}$ for wild-type $\mathrm{PHBH}^{20}$ as well as for the mutant enzymes. ${ }^{23}$ Buffers were made from nanopure-grade water (Millipore) and were filtered through a $0.22 \mu \mathrm{m}$ filter (Millipore). All chemicals used were of the highest purity available.

\subsection{Time-resolved fluorescence experiments}

Polarized time-correlated single-photon counting (TCSPC) was used to detect polarized time-resolved fluorescence on a (sub)nanosecond time scale. The instrumental setup for single-photon timing measurements have been described in detail earlier. ${ }^{1,2}$ Vertically polarized laser light of $450 \mathrm{~nm}$ or $460 \mathrm{~nm}$ was used to excite the sample with a frequency of $594 \mathrm{kHz}$ and duration of 4 ps FWHM. Parallel and perpendicularly polarized fluorescence was detected through $557.9 \mathrm{~nm}$ (for excitation at $450 \mathrm{~nm}$ ) or $526.0 \mathrm{~nm}$ (for excitation at $460 \mathrm{~nm}$ ) interference filters (Schott, Mainz, Germany) in combination with a kV 520 cut-off filter (Schott). Data were collected in a multichannel analyzer with a time window of 1024 channels with channel widths between 7-15 ps/ channel.

Analysis of the fluorescence intensity decay $I(t)$ was performed using the software package from Maximum Entropy Solutions Ltd. (Ely, United Kingdom), and the TRFA Data Processing package of the Scientific Software Technologies Center of 
Belarusian State University, Minsk, Belarus (www. sstcenter.com). ${ }^{41}$ With the maximum entropy method, the fluorescence intensity decays are described in terms of distributions of decay times, for which no a priori knowledge of the system is required. $^{42,43} \mathrm{~A}$ detailed description of the analysis procedures has been provided previously. ${ }^{1,2}$

\section{Results}

\subsection{Fluorescence quenching in $\mathrm{PHBH}$}

The experimental total-flavin fluorescence decay of uncomplexed wild-type PHBH exhibits a strongly heterogeneous character (figure 3). Although flavin fluorescence in PHBH is strongly quenched, the quenching is considerably less than in E. coli GR

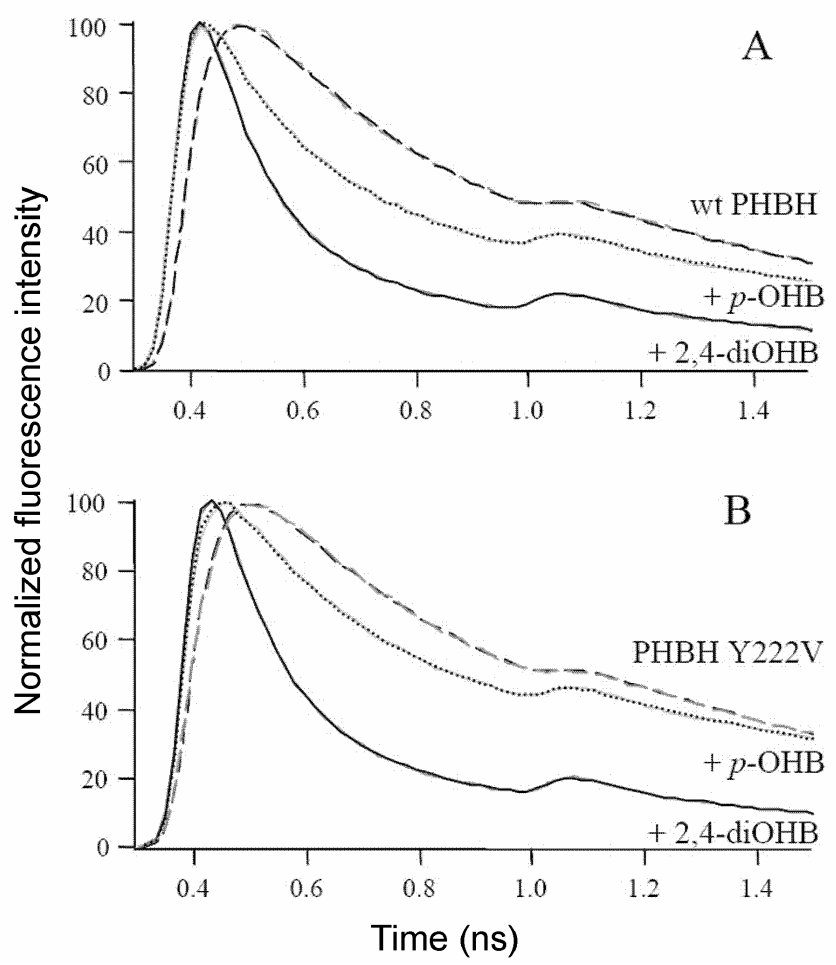

Figure 3. Experimental total fluorescence decays and corresponding theoretical data retrieved from the multiexponential fits of wild-type $\mathrm{PHBH}(\mathrm{A})$, and $\mathrm{PHBH}$ $\mathrm{Y} 222 \mathrm{~V}$ (B) in $50 \mathrm{mM}$ potassium phosphate buffer, $\mathrm{pH}$ 7.5 , at $298 \mathrm{~K}$, both free and in complex with $p$-OHB and 2,4-dihydroxybenzoate. Experimental data (grey) and theoretical data (black) overlay well. In order to clearly reveal the differences between the data, only the initial part of the time window is shown. The bumps after about $1 \mathrm{~ns}$ arise from a reflection artefact as described in ref. [2]. (the ratio of fluorescence quantum yields: $Q_{\mathrm{PHBH}} /$ $Q_{\mathrm{GR}}=15$ ). Inverse Laplace transformation with the MEM analysis method yielded for wild-type PHBH a pattern with five lifetime distributions covering the dynamic range from $\approx 10 \mathrm{ps}$ to $3 \mathrm{~ns}$ (figure 3 ). Predominant lifetime components were found near 15 , $0 \cdot 1$, and $0.6 \mathrm{~ns}$, and minor lifetime contributions near 1.5 and $3 \mathrm{~ns}$. Analysis with a sum of discrete exponentials confirmed the complex fluorescence lifetime behaviour of PHBH (table 2). Flavin fluorescence in the mutant enzymes PHBH Y222V and Y222A was somewhat less quenched than in wildtype enzyme. For analysis of the flavin fluorescence decays of the PHBH Y222 mutants, only four lifetime components were needed. The lifetimes of PHBH Y222V were nearly identical to the longer components observed for wild-type enzyme. For PHBH Y222A, the nanosecond component shifted to longer time constants and its relative amplitude increased. The picosecond lifetime component occurring in wild-type enzyme was not clearly resolved for both tyrosine mutants: a picosecond lifetime with low amplitude could be introduced, but only occasionally did it improve the quality of the fit. These results suggest that interaction with the Tyr222 causes the picosecond flavin fluorescence quenching process observed in wild-type enzyme. Fluorescence depolarization studies of wild-type $\mathrm{PHBH}$ and the tyrosine mutants did not reveal flavin mobility on the nanosecond time scale. The anisotropy decay was determined by rotational diffusion of the enzyme as a whole (for an example see figure 4).

\subsection{Fluorescence lifetime patterns of binary enzyme/substrate complexes}

In order to reveal possible specific fluorescence lifetime patterns for the 'in' and 'out' conformation of the FAD in $\mathrm{PHBH}$, fluorescence lifetime analysis was performed on binary enzyme/substrate complexes. For this, wild-type and Y222V PHBH were titrated with both $p$-OHB and 2,4-dihydroxybenzoate. As substrate binding in PHBH is known to depend on $\mathrm{pH}$ and solvent conditions, ${ }^{44}$ the dissociation equilibrium constants of the substrate analogues were determined from steady-state fluorescence quenching under the conditions used in time-resolved fluorescence analysis (table 3). Upon titration with the substrate analogues, the fluorescence lifetime distributions of wild-type and Y222V PHBH showed gradual changes. The lifetime distributions 
Table 2. Fluorescence lifetime parameters $\left(\tau_{i}, \alpha_{i}\right)$ of PHBH and its Y222 mutants, free and in complex with substrate analogues ${ }^{\mathrm{a}}$.

\begin{tabular}{|c|c|c|c|c|c|}
\hline \multicolumn{2}{|c|}{$\mathrm{PHBH}$} & \multicolumn{2}{|c|}{$\mathrm{PHBH} / p$-OHB complex ${ }^{\mathrm{b}}$} & \multicolumn{2}{|c|}{ PHBH/2,4-dihydroxybenzoate complex } \\
\hline$\tau_{i}(\mathrm{~ns})$ & $\alpha_{i}(-)$ & $\tau_{i}(\mathrm{~ns})$ & $\alpha_{i}(-)$ & $\tau_{i}(\mathrm{~ns})$ & $\alpha_{i}(-)$ \\
\hline $0.013 \pm 0.007$ & $0.40 \pm 0.09$ & $0.011 \pm 0.002$ & $0.84 \pm 0.05$ & $0 \cdot 015 \pm 0.005$ & $0.83 \pm 0.04$ \\
\hline $0.12 \pm 0.03$ & $0.27 \pm 0.07$ & $0 \cdot 15 \pm 0 \cdot 07$ & $0.09 \pm 0.03$ & $0.12 \pm 0.05$ & $0.13 \pm 0.02$ \\
\hline $0.61 \pm 0.05$ & $0.24 \pm 0.03$ & $0.76 \pm 0.10$ & $0 \cdot 04 \pm 0.02$ & $0.66 \pm 0.12$ & $0.03 \pm 0.01$ \\
\hline $1.65 \pm 0.06$ & $0.07 \pm 0.02$ & $2 \cdot 25 \pm 0.11$ & $0.03 \pm 0.01$ & $1.71 \pm 0.24$ & $0.01 \pm 0.01$ \\
\hline $3 \cdot 13 \pm 0.07$ & $0.02 \pm 0.00$ & - & - & - & - \\
\hline
\end{tabular}

\begin{tabular}{|c|c|c|c|c|c|}
\hline \multicolumn{2}{|c|}{ PHBH Y222V } & \multicolumn{2}{|c|}{$\mathrm{Y} 222 \mathrm{~V} / p-\mathrm{OHB}$ complex ${ }^{\mathrm{d}}$} & \multicolumn{2}{|c|}{ Y222V/2,4-dihydroxybenzoate complex } \\
\hline$\tau_{i}(\mathrm{~ns})$ & $\alpha_{i}(-)$ & $\tau_{i}(\mathrm{~ns})$ & $\alpha_{i}(-)$ & $\tau_{i}(\mathrm{~ns})$ & $\alpha_{i}(-)$ \\
\hline$-{ }^{f}$ & - & $0.010 \pm 0.003$ & $0.73 \pm 0.06$ & $0.013 \pm 0.005$ & $0.75 \pm 0.04$ \\
\hline $0.096 \pm 0.010$ & $0.34 \pm 0.01$ & $0 \cdot 10 \pm 0 \cdot 01$ & $0.15 \pm 0.01$ & $0.077 \pm 0.010$ & $0.21 \pm 0.02$ \\
\hline $0.57 \pm 0.01$ & $0.48 \pm 0.01$ & $0.63 \pm 0.30$ & $0.07 \pm 0.03$ & $0.53 \pm 0.07$ & $0.03 \pm 0.01$ \\
\hline $1.54 \pm 0.01$ & $0.15 \pm 0.01$ & $1.97 \pm 0.14$ & $0.04 \pm 0.02$ & $1.85 \pm 0.17$ & $0.01 \pm 0.00$ \\
\hline $2 \cdot 72 \pm 0.013$ & $0.03 \pm 0.01$ & $3 \cdot 37 \pm 0 \cdot 16$ & $0.01 \pm 0.00$ & - & - \\
\hline
\end{tabular}

\section{PHBH Y222A}

\begin{tabular}{lc}
\hline$\tau_{i}(\mathrm{~ns})$ & $\alpha_{i}(-)$ \\
\hline${ }^{\mathrm{f}}$ & - \\
$0 \cdot 094 \pm 0.010$ & $0 \cdot 30 \pm 0.02$ \\
$0.67 \pm 0.02$ & $0.43 \pm 0.01$ \\
$2 \cdot 28 \pm 0.02$ & $0.26 \pm 0.02$ \\
$4.95 \pm 0.20$ & $0.01 \pm 0.01$ \\
\hline
\end{tabular}

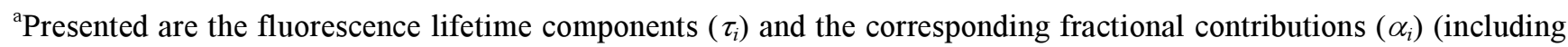
standard deviations) as obtained from the analysis of experiments performed in $50 \mathrm{mM}$ potassium phosphate buffer, $\mathrm{pH}$ 7.5, at $298 \mathrm{~K}$; ${ }^{\mathrm{b}}$ Saturation degree $\mathrm{PHBH} / p-\mathrm{OHB}$ : 91\%; ${ }^{\mathrm{c}}$ Saturation degree $\mathrm{PHBH} / 2,4$-dihydroxybenzoate: $91 \%$; ${ }^{\mathrm{d}} \mathrm{Satu}-$ ration degree PHBH Y222V/p-OHB: 84\%; ${ }^{\mathrm{e}}$ Saturation degree PHBH Y222V/2,4-dihydroxybenzoate: 94\%; ${ }^{\mathrm{f}}$ For the mutant enzymes PHBH Y222V and Y222A, a picosecond lifetime could not be resolved unambiguously

Table 3. Dissociation constants of complexes between $\mathrm{PHBH}(\mathrm{Y} 222 \mathrm{~V})$ and the substrates $p$-OHB and 2,4-dihydroxybenzoate ${ }^{\mathrm{a}}$.

\begin{tabular}{lccc}
\hline Enzyme & Ligand & Dissociation constant $^{\mathrm{a}}(\mathrm{mM})$ & Dissociation constant $^{\mathrm{b}}(\mathrm{mM})$ \\
\hline PHBH & $p$-OHB & 0.05 & 0.04 \\
PHBH & 2,4-Dihydroxybenzoate & $0 \cdot 10$ & 0.09 \\
PHBH Y222V & $p$-OHB & $1 \cdot 7$ & $1 \cdot 1$ \\
PHBH Y222V & 2,4-Dihydroxybenzoate & 0.06 & 0.07 \\
\hline
\end{tabular}

${ }^{a}$ Dissociation constants were determined fluorimetrically in $50 \mathrm{mM}$ potassium phosphate $\mathrm{pH} 7 \cdot 5$, at $298 \mathrm{~K}$. ${ }^{\mathrm{b}}$ Values are compared with dissociation constants in $100 \mathrm{mM}$ Tris/SO $/ \mathrm{pH} 8 \cdot 0$, taken from van der Bolt et al (1996)

at substrate concentrations near saturation are presented in figure 5 . The most striking effect from substrate binding is the emergence of an ultrashort fluorescence lifetime in all four enzyme/substrate complexes. Under saturating conditions, this direct substrate quenching dominates the lifetime patterns. Although in the titration experiments no full satura- tion was obtained, the sum of the amplitudes of the remaining longer lifetime components was higher than can be expected from the saturation degree if in every complex, fluorescence quenching occurs for $100 \%$ through ultrafast interaction with the bound substrate (table 2). If it is assumed that this effect is due to a small chance that flavin fluorescence is 
quenched through an interaction with other residues than the bound substrate, the longer lifetimes may reflect the 'in' or 'out' conformation of the flavin (depending on the enzyme-substrate combination used). All binary enzyme/substrate complexes studied, however, display contributions of longer lifetime components with time constants not far from the ones observed for the free enzymes (table 2; figure 5). Yet, the amplitude of the longer lifetimes seems to differ significantly for the specific combinations. Under the above-mentioned assumption, the 'in' and 'out' conformations of the flavin may thus be reflected in the ratios of the amplitude of the $0 \cdot 1-\mathrm{ns}$ component and that of the 0.6-ns (and 1.8-ns) component: for complexes with the flavin in the 'out' conformation (wt PHBH/2,4-dihydroxybenzoate and PHBH Y222V/2,4-dihydroxybenzoate), a relatively larger contribution of the $0 \cdot 1$ ns component is observed, whereas in complexes in which one may expect the 'in' conformation (wt $\mathrm{PHBH} / p$-OHB), the amplitudes of the longer lifetime components are somewhat more equal. We shall further address this issue in the discussion section.

\section{Discussion}

In contrast with glutathione reductase and thioredoxin reductase, $\mathrm{PHBH}$ has an active-site region that can accommodate substantial movements of the flavin ring. Crystallographic and kinetic data have suggested that in PHBH this flavin mobility is es-

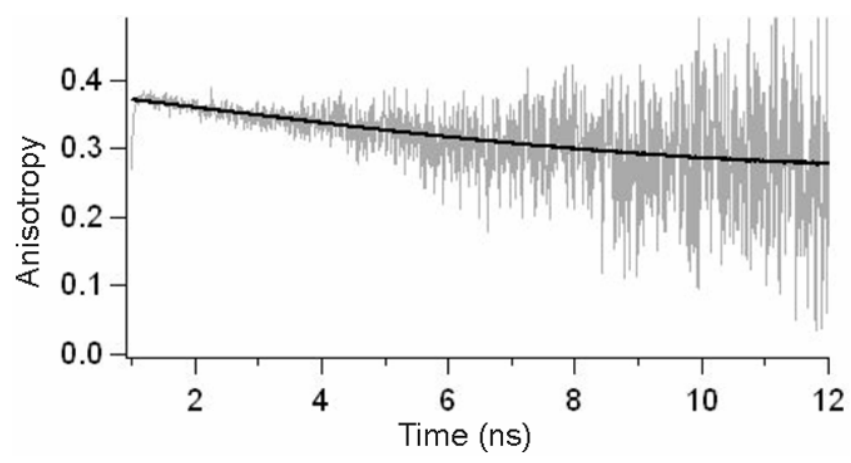

Figure 4. Experimental fluorescence anisotropy decay (grey) and corresponding theoretical curve (black) retrieved from the mono-exponential fit of $4 \mu \mathrm{M}$ wild-type $\mathrm{PHBH}$ in $50 \mathrm{mM}$ potassium phosphate buffer, $\mathrm{pH} 7 \cdot 5$, at $293 \mathrm{~K}$. Experimental and theoretical data overlay well. The recovered parameters are the initial anisotropy $r(0)=0.37$ and the rotational correlation time $\phi=32 \pm$ 2 ns. A rotational correlation time of 32 ns agrees well with a protein of $90 \mathrm{kDa}$. sential for catalysis: whereas the flavin in the 'in' position provides a suitable environment for efficient hydroxylation of the substrate (shielded from the solvent), the swinging 'out' of the flavin creates a pathway for substrate binding and product release, and facilitates efficient flavin reduction. It has been suggested that mobility of the flavin ring also plays an important role in the catalysis of the related enzyme phenol hydroxylase. ${ }^{45}$ Optimal regulation of catalysis in these redox enzymes requires strict regulation of the cofactor mobility. Specific (transient) interactions with particular amino acids as well as interactions with the substrate can contribute to this regulation. These interactions, however, also determine the photophysical characteristics of the flavin fluorophore.

For substrate-free $\mathrm{PHBH}$, this is shown in the fluorescence lifetime data of the Tyr222 mutants. Here, we have demonstrated that this residue invol-
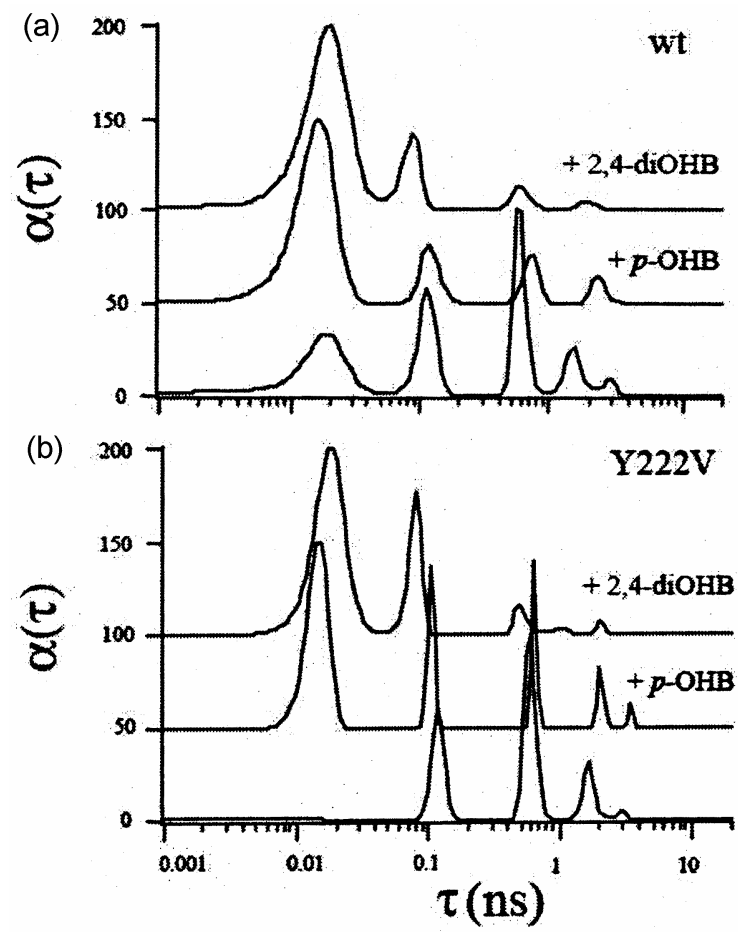

Figure 5. Normalized fluorescence lifetime distributions of wild-type PHBH (a) and PHBH Y222V (b) uncomplexed and in complex with $p$-OHB and 2,4-dihydroxybenzoate in $50 \mathrm{mM}$ potassium phosphate buffer, $\mathrm{pH}$ $7 \cdot 5$, at $298 \mathrm{~K}$. Binary enzyme-substrate complexes were formed under (nearly) saturating conditions resulting in percentage of complex: $\mathrm{PHBH} / p$-OHB: 91\%; $\mathrm{PHBH} / 2,4-$ dihydroxybenzoate: 91\%; PHBH Y222V/p-OHB: 84\%; PHBH Y222V/2,4-dihydroxybenzoate: 94\%. For clarity, a vertical offset has been applied. 
Table 4. Shortest distance between various potential quenching sites and the isoalloxazine ring of FAD in wild-type $\mathrm{PHBH}$ in the 'in' and 'out' conformations $\mathrm{a}^{\mathrm{a}}$.

\begin{tabular}{lccc}
\hline Residue & Conformation & Flavin atom & $\AA$ \\
\hline Trp185-CZ3 & In & C6 & $5 \cdot 1$ \\
& Out & O4 & $5 \cdot 2$ \\
Tyr201-OH & In & O4 & $6 \cdot 7$ \\
& Out & O 2 & $8 \cdot 1$ \\
Tyr222-OH & In & C6 & $3 \cdot 9$ \\
& Out & O4 & $3 \cdot 3$ \\
Tyr385-OH & In & O4 & $6 \cdot 9$ \\
& Out & O2 & $8 \cdot 7$ \\
\hline
\end{tabular}

${ }^{a}$ Distances were retrieved from the PHBH- $p$-OHB complex resolved at $1.9 \AA$ resolution for the 'in' conformation, and from the PHBH-2,4-dihydroxybenzoate complex resolved at $2 \cdot 5 \AA$ resolution for the 'out' conformation (Schreuder et al 1994)

ved in controlling flavin mobility, can efficiently quench flavin fluorescence. Both in the 'in' and 'out' conformation Tyr222 is located within $4 \AA$ from the isoalloxazine ring (table 4). Based on the similarity in structure and fluorescence characteristics between PHBH and other tyrosine-containing flavoenzymes such as glutathione reductase, ${ }^{1}$ NADPH peroxidase ${ }^{36}$ and glucose oxidase, ${ }^{46-48}$ the mechanism of quenching most probably involves photoinduced electron transfer from this particular tyrosine to the light-excited flavin (see also ref. [37]). The considerably lower amplitude of ultrafast quenching of PHBH in comparison with the enzymes mentioned above may reflect the increased freedom of the flavin cofactor: in the fraction of enzyme molecules in which close contact exists between the properly oriented flavin and tyrosine at the moment of excitation, ultrafast fluorescence quenching can occur. In the fraction of enzyme molecules responsible for the longer fluorescence lifetime components, quenching occurs through interactions with other residues, in a similar manner as observed for the PHBH Y222 mutants (this refers to the so-called multiple quenching sites $\operatorname{model}^{1,49}$ ). Based on the distance to the flavin, Trp185 as well as Tyr201 and Tyr385 (the latter two residues are both involved in substrate binding) may also contribute to fluorescence quenching. From comparison with fluorescence quenching characteristics of other flavoenzymes, a position adjacent to the more polar parts of the isoalloxazine ring, particularly near the electrophilic sites at $\mathrm{N} 4$ and $\mathrm{C} 4 \mathrm{a}$, is expected to result in more efficient fluorescence quenching than location near the relatively apolar dimethyl substituted ring (for discussion see ref. [3]). For Tyr222, it can thus be speculated that not only the distance, but also the relative position with respect to the flavin in the 'out' conformation is better suited for efficient fluorescence quenching than in the 'in' conformation (table 4). A more favourable orientation for quenching in the 'out' conformation may also be the case for Trp185. For Tyr201 and Tyr385, however, the distance to the flavin is somewhat shorter in the 'in' conformation. On the whole, the quenching characteristics of the flavin microenvironment do not drastically change from the 'in' to the 'out' conformation, so that the above-mentioned sites may well contribute to fluorescence quenching in both conformations, and no unique fluorescence lifetime pattern is observed.

For the isoalloxazine ring in the oxidized state, significant deviations from planarity are not expected to occur, irrespective of the protonation state or hydrogen bonding environment of the flavin. ${ }^{50}$ As this study is limited to the oxidized state, distances and orientations should therefore not be influenced much by mobility within the flavin ring. It should be kept in mind, however, that distances and orientations were retrieved from crystal structures of enzyme/substrate complexes. Though crystallographic studies of the free enzyme show the isoalloxazine ring in an intermediate position, ${ }^{33}$ it cannot be excluded that -in particular in the uncomplexed enzyme - the flavin ring adopts positions beyond the ones observed in the enzyme/substrate complexes, reducing the latter to only 'intermediate' conformations. The conformations in crystal structures should therefore be regarded as snapshots, of which the observed extremes have been defined as 'in' and 'out'. Though fluorescence can (simultaneously) sample all 'real' conformations in solution, experiments on flavin systems are in general limited to the oxidized state due to the low intrinsic fluorescence and complex photophysical and photochemical behaviour of the flavin in the two-electron reduced state. Interpretation of fluorescence characteristics in terms of dynamics in complex biological systems using twoelectron reduced flavin is still a bridge too far. This reduces the window of the reaction pathway of PHBH accessible with ensemble-detected fluorescence spectroscopy to the very first step: substrate binding in the oxidized state. From the point of view of dynamics, however, this step is particularly interesting as it necessarily involves significant mobility 
of the flavin. In addition, other than a possible bending of the isoalloxazine ring in the two-electron reduced state ${ }^{50,51}$ no indications exist that for completing the reaction cycle, conformations are required that are not in between the 'in' and 'out' conformation (including intermediate ones) as can be sampled in titration studies with different substrate analogues.

Aromatic substrate binding most efficiently induces flavin fluorescence quenching. As the substrates bind at van der Waals distance of the redox-active flavin, quenching here may also occur through photoinduced electron transfer. The quenching effects of $p$-hydroxybenzoate derivatives on the fluorescence lifetime distribution of PHBH also strengthen the multiple quenching sites model. Although saturation was not fully reached, the sum of amplitudes of the remaining longer lifetime components was higher than can be expected from the saturation degree (see $\$ 3$ ). It is therefore likely that in the complex there is still a small chance that flavin fluorescence is quenched through an interaction with an active-site residue. A less likely explanation is that through insufficient time resolution, the amplitude of the ultrafast component is underestimated and/or even faster fluorescence lifetimes were not resolved. Although our experiments with a lower time resolution of 5-10 ps do not rule out such effect, they do not explain the differences in amplitude of the resultant longer lifetimes between complexes in the 'in' conformation and those expected to have the 'out' conformation (see \$3). A good test model as to whether the relative amplitudes of the resultant longer lifetimes indeed reflect the flavin conformation is the $\mathrm{PHBH} \mathrm{Y} 222 \mathrm{~V} / p-\mathrm{OHB}$ complex, for which on the basis of absorption difference spectra ${ }^{23}$ and similarity with mutant PHBH Y222 $\mathrm{F}^{5}$ a predominant 'out' conformation is expected. Taking into account that the saturation degree obtained for this complex is lower than for the other enzyme/ substrate complexes so that a larger contribution of the ligand-free enzyme (16\%) shows up, a (partly) 'out' conformation of the flavin can indeed be deduced. The lifetime spectrum of this complex showed a ratio for the amplitudes of the 0.1- and 0.6-ns components of $\alpha_{0.1} / \alpha_{0.6}=2$. As the $0.6 \mathrm{~ns}$ component has the largest amplitude in the lifetime spectrum of free enzyme, the lifetime spectrum of $100 \%$ complex should therefore contain significantly larger amplitude for the 0.1-ns component. It can thus be concluded that the relative contributions of the longer lifetime components indeed correlate with the presence of the flavin conformation. Owing to the predominant fluorescence quenching by aromatic substrates together with the combined geometrical and photophysical characteristics of the active-site, lifetime detection is not a very sensitive method to probe the flavin conformation in $\mathrm{PHBH}$.

(Sub)nanosecond resolved fluorescence anisotropy detection failed to reveal direct mobility of the flavin cofactor (see figure 4). The absence of a fast, nanosecond relaxation process observed in the fluorescence anisotropy of PHBH as observed in glutathione reductase $\mathrm{e}^{2}$ and NADPH peroxidase ${ }^{36}$ and ascribed to a transient charge-transfer interaction between light-excited flavin and tyrosine, also indicates that the relative orientation of Tyr222 is unfavorable to exhibit this effect. A likely explanation for the lack of internal motion is that flavin mobility (from the 'in' to a more 'out' conformation and vice versa) occurs on a much slower time scale e.g. of the order of microseconds. Single-molecule fluorescence correlation spectroscopy on PHBH labelled with an Alexa dye indeed showed the presence of a relaxation time of $23 \mu \mathrm{s}$ during catalysis that is connected to the equilibrium of PHBH in oxidized and reduced forms. ${ }^{40}$ The inverse of the relaxation time is equal to the relaxation rate constant $\left(44 \times 10^{3} \mathrm{~s}^{-1}\right)$, which is a thousand times larger than the turnover rate $\left(37 \mathrm{~s}^{-1}\right)$ of the enzyme. ${ }^{38}$ It is nonetheless tempting to associate this relaxation process to the interconversion of the flavin between 'in' and 'out' conformations, since comparable conformational flexibility with characteristic relaxation times of $1-40 \mu$ s were reported for other proteins using FCS. ${ }^{52,53}$

A serious complication for monitoring the conformation and mobility of the flavin cofactor in PHBH with ensemble-detecting techniques in general, is that it is impossible to obtain data (free from direct influence of the substrate) solely from one conformation: as the flavin in the substrate-free PHBH is flexible, ${ }^{33}$ ensemble data of the substratefree enzyme shall contain a mixture of 'in', 'out' and/or 'intermediate' characteristics, dependent on the equilibrium conditions. Complex formation with a substrate to trap the flavin in a single conformation is therefore a necessary but potential risky method that may have direct impact on the interpretation of ensemble data of PHBH. This may also apply to other techniques such as Raman spectroscopy. ${ }^{54} \mathrm{~A}$ way to overcome such complication is to monitor 
flavin mobility in a single substrate-free $\mathrm{PHBH}$ molecule. By registering a sufficiently long time trace of a single enzyme molecule, we expect to cover the conformational space of the flavin, thereby acquiring specific characteristics of each of the conformational states encountered. These single-molecule studies have been recently reported for wildtype and Y222A-PHBH in the absence of substrates. ${ }^{55}$ The single-molecule fluorescence trajectories of substrate-free PHBH indeed reveal data that are consistent with the movement of the isoalloxazine between the less fluorescent 'in' conformation and a more fluorescent 'out' conformation. Interestingly, and in line with our results, the Y222A mutant was more fluorescent and did not fluctuate in the single-molecule trajectories. Statistical analysis of the interconversion in wild-type PHBH shows for the out-to-in transition a rate constant of $6.4 \mathrm{~s}^{-1}$ and for the in-to-out transition a rate constant of $21.0 \mathrm{~s}^{-1}$ yielding an equilibrium constant of 3.3 favouring the exposed state ${ }^{55}$ By using single-molecule measurements only one immobilized enzyme molecule is watched during a period of $30 \mathrm{~s}$ with a time resolution of 10 milliseconds. These experiments have been repeated for one hundred other single enzyme molecules enabling statistical analysis that leads to rates, which values are comparable to turnover rates. In the single-photon timing experiments millions of events of many enzyme molecules are monitored during 50-ns periods, much shorter than the real catalytic events. Information is obtained on 'pseudo' stationary states of the enzyme.

\section{Concluding remarks}

The fluorescence lifetime distribution of FAD in PHBH exhibits a complex pattern indicating fluorescence quenching-induced by multiple amino acids. The main objective of these experiments was to identify these quenching residues and to disentangle quenching mechanisms. Notwithstanding its complexity the four main findings and conclusions can be summarized as follows. First, we have shown that mutating Y222 into inert amino acids such as alanine or valine makes the lifetime distribution simpler by removing the ultrafast $10-15$ ps lifetime component. Second, binding of substrates to mutant or wild-type enzymes reintroduces or emphasizes the ultrafast 10-15 lifetime component indicating efficient quenching of the FAD fluorescence by photoinduced electron transfer from bound substrate to light- excited flavin. Third, we have discussed that these ensemble experiments do not allow the observation of separate conformations but, depending on the equilibrium conditions, the experimental data rather monitor a mixture of conformations ('in', 'out' or 'intermediate') that do not interconvert on the nanosecond timescale. Fourth, we have discussed the experimental findings in relation to recently published single-molecule fluorescence experiments. We conclude that the experiments on PHBH immobilized in an agarose matrix have too slow time resolution to resolve conformational relaxation processes, but can distinguish both conformations because the 'in' conformation shows quenched flavin fluorescence as compared to the 'out' conformation. ${ }^{55}$ On the other hand, experiments using fluorescence correlation spectroscopy on labelled PHBH in aqueous solution have sufficient time resolution to actually measure these relaxation times. ${ }^{40}$

\section{Acknowledgements}

We thank Dr F J T van der Bolt and Dr M H M Eppink for the construction of the mutant enzymes and helpful practical suggestions. The Netherlands Organisation for Scientific Research is thanked for financial support

\section{References}

1. van den Berg $\mathrm{P} A \mathrm{~A}$, van Hoek $\mathrm{A}$, Walentas $\mathrm{C} \mathrm{D}$, Perham R N and Visser A J W G 1998 Biophys. J. 74 2046

2. van den Berg P A W, van Hoek A and Visser A J W G 2004 Biophys. J. 872577

3. van den Berg P A W, Mulrooney S B, Gobets B, van Stokkum I H M, van Hoek A, Williams C H and Visser A J W G 2001 Protein Sci. 102037

4. Schreuder H A, Mattevi A, Obmolova G, Kalk K H, Hol W J G, van der Bolt F J T and van Berkel W J H 1994 Biochemistry 3310161

5. Gatti D L, Palfey B A, Lah M S, Entsch B, Massey V, Ballou D P and Ludwig M L 1994 Science 266 110

6. van Berkel W J H, Kamerbeek N M and Fraaije M W 2006 J. Biotechnol. 124670

7. Stanier R Y and Ornston L N $1973 A d v$. Microb. Physiol. 989

8. Entsch B and van Berkel W J H 1995 FASEB J. 9476

9. Entsch B, Cole L J and Ballou D P 2005 Arch. Biochem. Biophys. 433297

10. Husain M and Massey V 1979 J. Biol. Chem. 254 6657

11. Entsch B, Palfey B A, Ballou D P and Massey V 1991 J. Biol. Chem. 26617341 
12. Eschrich $\mathrm{K}$, van der Bolt $\mathrm{F} \mathrm{J} T$, de Kok $A$ and van Berkel W J H 1993 Eur. J. Biochem. 216137

13. Palfey B A, Moran G R, Entsch B, Ballou D P and Massey V 1999 Biochemistry 381153

14. Entsch B, Ballou D P and Massey V $1976 \mathrm{~J}$. Biol. Chem. 2512550

15. Spector T and Massey V 1972 J. Biol. Chem. 247 4679

16. Wierenga R K, de Jong R J, Kalk K H, Hol W G J and Drenth J 1979 J. Mol. Biol. 13155

17. Schreuder H A, van der Laan J M, Hol W G J and Drenth J 1988 J. Mol. Biol. 199637

18. Schreuder H A, Prick P, Wieringa R K, Vriend G, Wilson K S, Hol W G J and Drenth J $1989 \mathrm{~J}$. Mol. Biol. 208679

19. Schreuder H A, van der Laan J M, Swarte M B A, Kalk K H and Hol W G J 1992 Proteins: Struct. Funct. Genet. 141178

20. van Berkel W J H, Westphal A H, Eschrich K, Eppink M H M and de Kok A 1992 Eur. J. Biochem. 210411

21. van Berkel W J H, van der Bolt F J T, Eppink $M H$ M, de Kok A, Rietjens I M C M, Veeger C, Vervoort $\mathrm{J}$ and Schreuder H A 1994 in Flavins and flavoproteins $X I$ (ed.) K Yagi (Berlin: Walter de Gruyter) p. 231

22. Moran G R, Entsch B, Palfey B A and Ballou D P 1999 Biochemistry 386292

23. van der Bolt F J T, Vervoort J and van Berkel W J H 1996 Eur. J. Biochem. 237592

24. van der Laan J M, Schreuder H A, Swarte M B A, Wierenga R K, Kalk K H, Hol W G J and Drenth J 1989 Biochemistry 287199

25. Schreuder H A, van der Laan J M, Hol W G J and Drenth J 1991 in Chemistry and biochemistry of flavoenzymes (ed.) F Müller (Boca Raton: CRC Press) p. 31

26. Eppink $\mathrm{M} \mathrm{H}$, Schreuder $\mathrm{H} A$ and van Berkel W J 1998 J. Biol. Chem. 27321031

27. Wang J, Ortiz-Maldonado M, Entsch B, Massey V, Ballou D and Gatti D L 2002 Proc. Natl. Acad. Sci. USA 99608

28. Eppink M H, Schreuder H A and Van Berkel W J H 1995 Eur. J. Biochem. 231157

29. Eppink M H, Schreuder H A and van Berkel W J H 1998 Eur. J. Biochem. 253194

30. Eppink M H, Overkamp K M, Schreuder H A and Van Berkel W J H 1999 J. Mol. Biol. 29287

31. van Berkel W J H, Eppink M H M and Schreuder H A 1994 Protein Sci. 32245

32. Palfey B A, Ballou D P and Massey V 1997 Biochemistry 3615713

33. Eppink M H M, van Berkel W J H, Teplyakov A and Schreuder H A 1999 in Flavins and flavoproteins (eds) S Ghisla, P Kroneck, P Macheroux and H Sund (Berlin: Agency for Sci. Publications) p. 239

34. Ortiz-Maldonado M, Gatti D, Ballou D P and Massey V 1999 Biochemistry 3816636

35. Ballou D P, Entsch B and Cole L J 2005 Biochem. Biophys. Res. Commun. 338590

36. Visser A J W G, van den Berg P A W, Visser N V, van Hoek $A$, van den Burg $H$ A, Parsonage D and Claiborne A 1998 J. Phys. Chem. B102 10431

37. van den Berg P A W and Visser A J W G 2001 in New trends in fluorescence spectroscopy. Applications to chemical and life sciences (eds) B Valeur and J C Brochon (Berlin: Springer) p. 457

38. van Berkel W J H and Müller F 1987 Eur. J. Biochem. 16735

39. Eschrich $\mathrm{K}$, van Berkel W J H, Westphal A H, de Kok A, Mattevi A, Obmolova G, Kalk K H and Hol W G J 1990 FEBS Lett. 277197

40. Westphal A H, Matorin A, Hink M A, Borst J W, van Berkel W J H and Visser A J W G $2006 \mathrm{~J}$. Biol. Chem. 28111074

41. Digris A V, Skakoun V V, Novikov E G, van Hoek A, Claiborne A and Visser A J W G 1999 Eur. Biophys. J. 28526

42. Livesey A K and Brochon J C 1987 Biophys. J. 52 693

43. Brochon J C 1994 Methods Enzymol. 240262

44. van Berkel W J H and Müller F 1989 Eur. J. Biochem. 179307

45. Enroth C, Neujahr H, Schneider G and Lindqvist $Y$ 1998 Structure 6605

46. Mataga N, Chrosrowjan H, Shibata Y and Tanaka F 1998 J. Phys. Chem. B102 7081

47. Mataga N, Chrosrowjan H, Shibata Y, Tanaka F, Nishina Y and Shiga K 2000 J. Phys. Chem. B104 10667

48. Zhong D and Zewail A H 2001 Proc. Natl. Acad. Sci. USA 9811867

49. Bajzer Z and Prendergast F G 1993 Biophys. J. 65 2313

50. Zheng Y J and Ornstein R L $1996 \mathrm{~J}$. Am. Chem. Soc. 1189402

51. Hall L H, Orchard B J and Tripathy S K 1987 Int. J. Quantum Chem. 31195

52. Chattopadhyay K, Elson E L and Frieden C 2005 Proc. Natl. Acad. Sci. USA 1022385

53. Neuweiler H, Doose S and Sauer M 2005 Proc. Natl. Acad. Sci. USA 10216650

54. Zheng Y, Dong J, Palfey B A and Carey P R 1999 Biochemistry 3816727

55. Brender J R, Dertouzos J, Ballou D P, Massey V, Palfey B A, Entsch B, Steel D G and Gafni A $2005 \mathrm{~J}$. Am. Chem. Soc. 12718171 